\title{
ARTEFATOS BIOLÓGICOS NO EEG QUANTITATIVO
}

\author{
Renato Anghinah ${ }^{1,2}$, Luis I. Basile ${ }^{1,3}$, Magali T. Schmidt', \\ Koichi Sameshima', Wagner Farid Gattaz ${ }^{1}$
}

\begin{abstract}
RESUMO - Estudamos, em 10 indivíduos adultos normais, o comportamento de cinco artefatos biológicos do eletrencefalograma (EEG): piscamento palpebral, fechamento forçado dos olhos, fechamento forçado da mandíbula, movimentos de língua e varredura horizontal dos olhos - tanto por análise visual como espectral - tanto com objetivo de verificar como esses artefatos são visualizados quando apresentados em mapas de potência da amplitude espectral. Observamos que os potenciais do espectro respeitavam a mesma disposição topográfica que os encontrados à análise visual do traçado. A análise visual do EEG é superior à quantitativa, para o reconhecimento de artefatos, porque preserva a visualização morfológica dos grafoelementos que deve ser feita obrigatoriamente no domínio do tempo, pois a sua correta identificação se perde no domínio da frequência. Devido a grande dificuldade de excluirmos totalmente os artefatos durante o registro do EEG e, por conseguinte, serem incluídos na análise quantitativa, é fundamental conhecermos como estes potenciais serão representados nos mapas quantitativos, para podermos identificalos, evitando confundí-los com atividades patológicas do EEG.
\end{abstract}

PALAVRAS-CHAVE: artefatos, eletrencefalograma, mapeamento cerebral.

\section{Biologic artifacts in quantitative EEG}

\begin{abstract}
We studied the influence of five biologic artifacts sources on quantitative EEG (blinking, forced eyes closure, forced jaw closure, tongue movements and pursuit eyes movements) through both visual and spectral analysis, with the purpose of verifying how do these artifacts can be seen in a cartographic way. We found that the spectrum's potencials showed the same topographic display that was found through visual analysis. Visual analysis was superior than the quantitative evaluation to recognise the artifacts, as the former preserved the morphological display of the paroxisms. However it is important know how do the potencials are represented in quantitative maps, so that they can be identified as artifacts and not as pathologic EEG activity.
\end{abstract}

KEY WORDS: artifacts, electroencephalogram, brain mapping.

A rtefatos em eletrencefalografia (EEG) são definidos como todo potencial elétrico proveniente de outra fonte que não seja o cére b ro'. É raro um traçado de EEG que não tenha artefatos, a redução dependendo de uma técnica apurada do eletrencefalogra fista e da colaboração do paciente ${ }^{1,2}$. Os artefatos p rovocados de ocorrência mais comum são os instrumentais, como os de eletrodo e fisiológicos ou biológicos. Estes incluem os movimentos oculares, musculares, respiratórios entre outros ${ }^{2-5}$.

Os artefatos podem alterar substancialmente os resultados de análise espectral e a disposição topográfica. Depois de processados, eles não podem ser eliminados. Deste modo, o melhor método para evi- tarmos os artefatos no EEG quantitativo e topográfico é identificando-os durante a análise visual do traçado, evitando incluí-los nos trechos a serem processadas pela transformada rápida de Fourier ${ }^{5,6}$. Esta pratica é recomendada fortemente pelos textos especializados no assunto ${ }^{7}$. Porém, muitas vezes torna-se praticamente impossível, mesmo ao mais experiente eletrencefalografista, selecionar para a análise quantitativa trechos do traçado absolutamente livres de artefatos $^{8,9}$. Portanto, conhecer a sua apresentação após o processo de quantificação é recomendável, para que não sejam interpretados de maneira errônea.

O objetivo deste trabalho foi estudar o comportamento de cinco artefatos fisiológicos (piscamento pal-

\footnotetext{
'Laboratório de Neurociências (LIM 27) Instituto de Psiquiatria do Hospital das Clínicas (HC) da Faculdade de Medicina da Universidade de São Paulo (FMUSP), São Paulo SP, Brasil; ${ }^{2}$ G rupo de Neurologia Cognitiva e do Comportamento do HC-FMUSP; ${ }^{3}$ Faculdade de Psicologia e Fonoaudiologia, Universidade Metodista.
}

Recebido 1 Setembro 2005, recebido na forma final 21 Novembro 2005. Aceito 18 Janeiro 2006. 


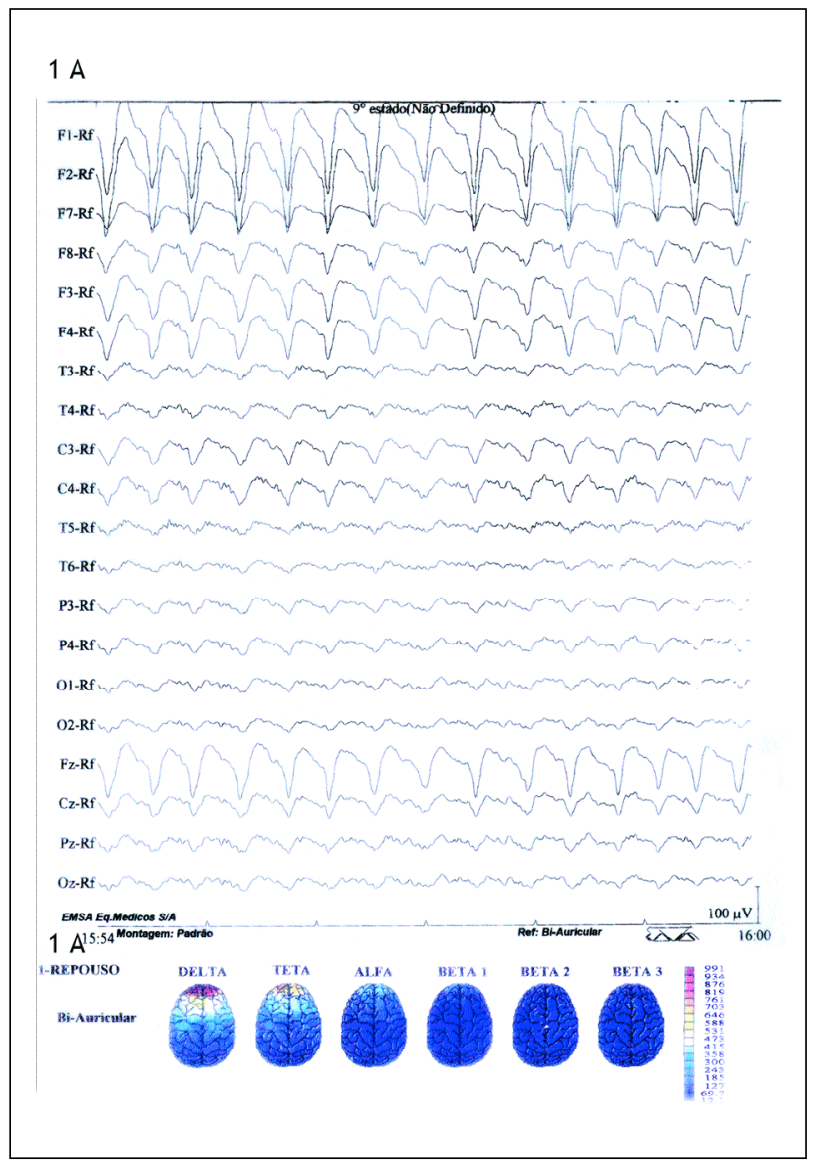

Fig 1. Piscamento palpebral. A) análise visual do EEG: ondas delta de alta amplitude e polaridade negativa em eletrodos Fp1 e Fp2. B) análise espectral: maiores amplitudes do espectro em regiões anteriores bilateralmente, nas bandas delta e teta.

pebral, fechamento forçado dos olhos, fechamento forçado da mandíbula, movimentos de língua e varredura horizontal dos olhos) quando representados na fo rma cartográfica, comparados a análise visual.

\section{MÉTODO}

Realizamos registros de EEG em repouso e olhos fechados durante 30 minutos, em 6 homens e 4 mulheres voluntários,com idades entre 22 a 41 anos (media 27 anos), sem queixas clínicas e exames clínico e laboratoriais normais. Utilizamos equipamento de EEG quantitativo da marca EMSA, com 20 canais, processador de 12 bits e freqüência amostral de 256 pontos, eletrodos de escalpo, com impedância menor que 10 kohms e montagem obedecendo o sistema $10-20^{10-13}$.

Todos os participantes assinaram o consentimento livre escla recido e o estudo foi aprovado pelo conselho de ética por fazer parte do piloto de estudo mais amplo clínicolaboratorial do LIM 27 IPq FMUSP.

Durante o exame, os pacientes foram solicitados a exe-

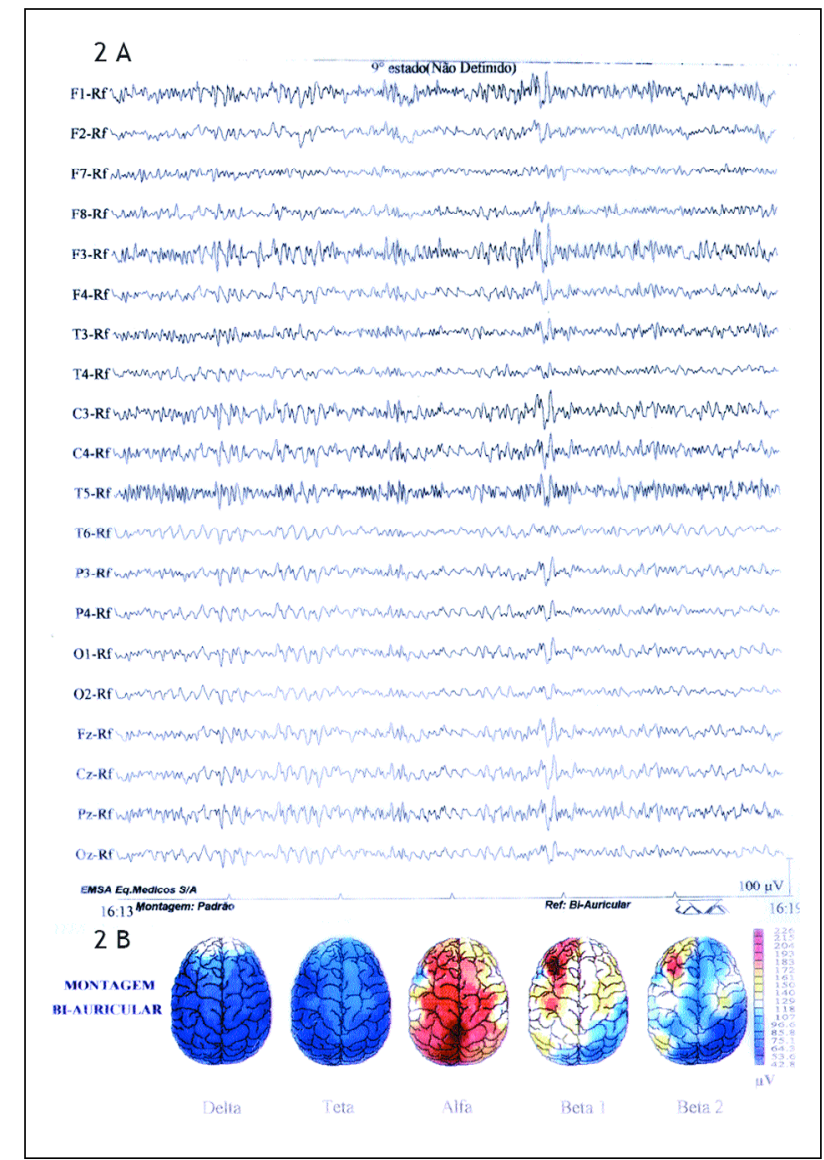

Fig 2. Fechamento forçado dos olhos. A) análise visual do EEG: atividade rápida (padrão muscular) Fp1 e Fp2. B) análise espec tral: maiores ampltudes espectrais nas bandas alfa (predomínio posterior) e nas bandas beta 1 e beta 2 (regiões anteriores e médias).

cutar movimentos de piscamento palpebral, fecham ento forçado dos olhos, fechamento forçado da mandíbula, movimentos de língua e movimentação de varredura dos olhos por cerca de 30 segundos a 1 minuto, cada um deles. Após isto, selecionamos por exame, de 5 a 10 épocas de 2,56 segundos para cada tipo de artefato.

\section{RESULTADOS}

Todos os exames foram normais à análise visual e quantitativa.

Os achados tanto à análise visual como quantitativa e topográfica do EEG obedeceram sempre aos mesmos padrões para cada tipo de artefato, que foram:

Piscamento palpebral - A análise visual de EEG mostrou ondas delta de alta amplitude e polaridade negativa, monofásicas, padrão regular com frequência ao redor de $2,0 \mathrm{~Hz}$ localizadas em eletrodos Fp1 e Fp2, com difusão para eletrodos F7-F8 e F3-F4. A 


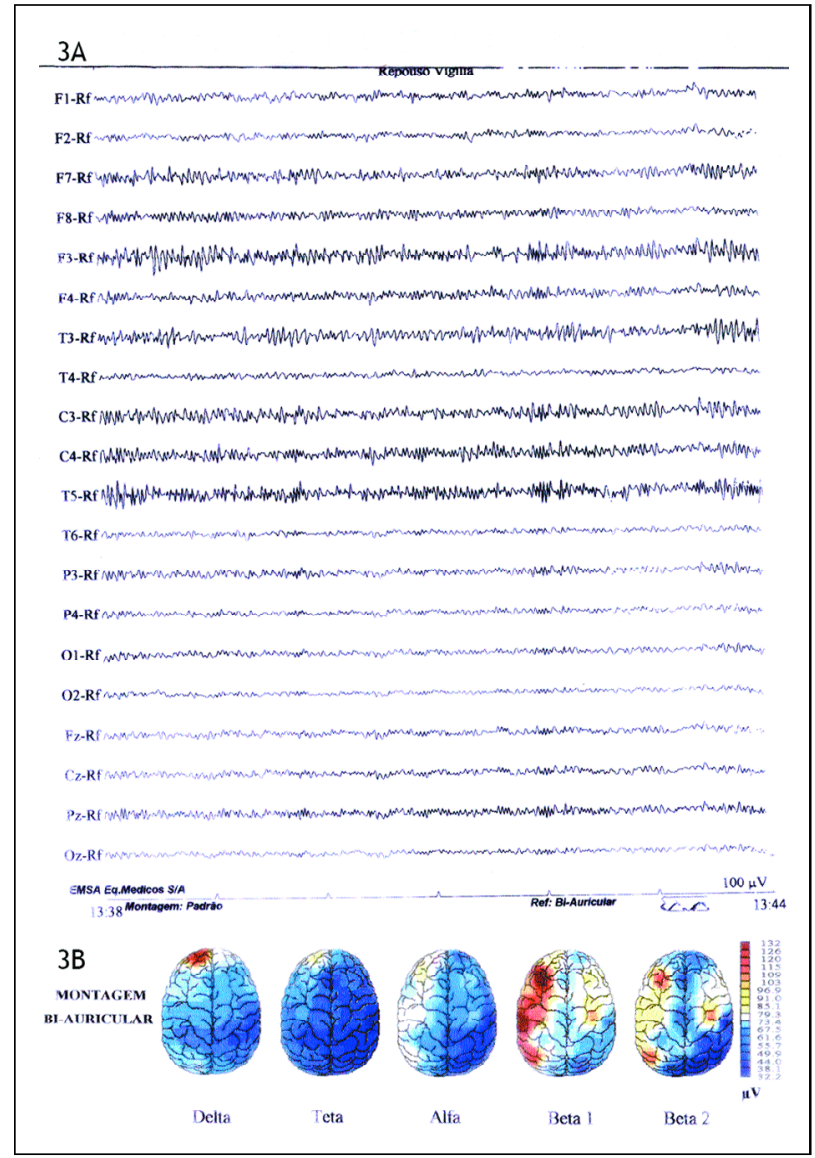

Fig 3. Fechamento forçado da mandíbula. A) análise visual do EEG: atividade rápida em regiões temporais anteriores e pos teriores. B) análise espectral: maiores amplitudes do espectro em região temporal média bilateralmente nas faixas beta 1 e beta 2 .

quantificação destes achados mostrou distribuição espectral com maiores amplitudes em regiões anteriores bilateralmente, nas bandas deltas (predominando em 3,52 Hz) e teta (Fig 1).

Fechamento forçado dos olhos - A análise visual do EEG mostrou atividade rápida (padrão muscular) captadas em eletrodos Fp1 e Fp2. A quantificação destes achados mostrou a presença de maiores amplitudes nas faixas de freqüência alfa de predomínio posterior (paciente em vigília e olhos fechados), além de amplitude espectral aumentada em regiões anteriores e médias na faixa de freqüência beta 1 (predominante) e beta 2. Observamos também na banda delta, um p redomínio espectral em regiões anteriores (Fig 2).

Fechamento forçado da mandíbula - A análise visual do EEG mostrou uma atividade rápida (padrão

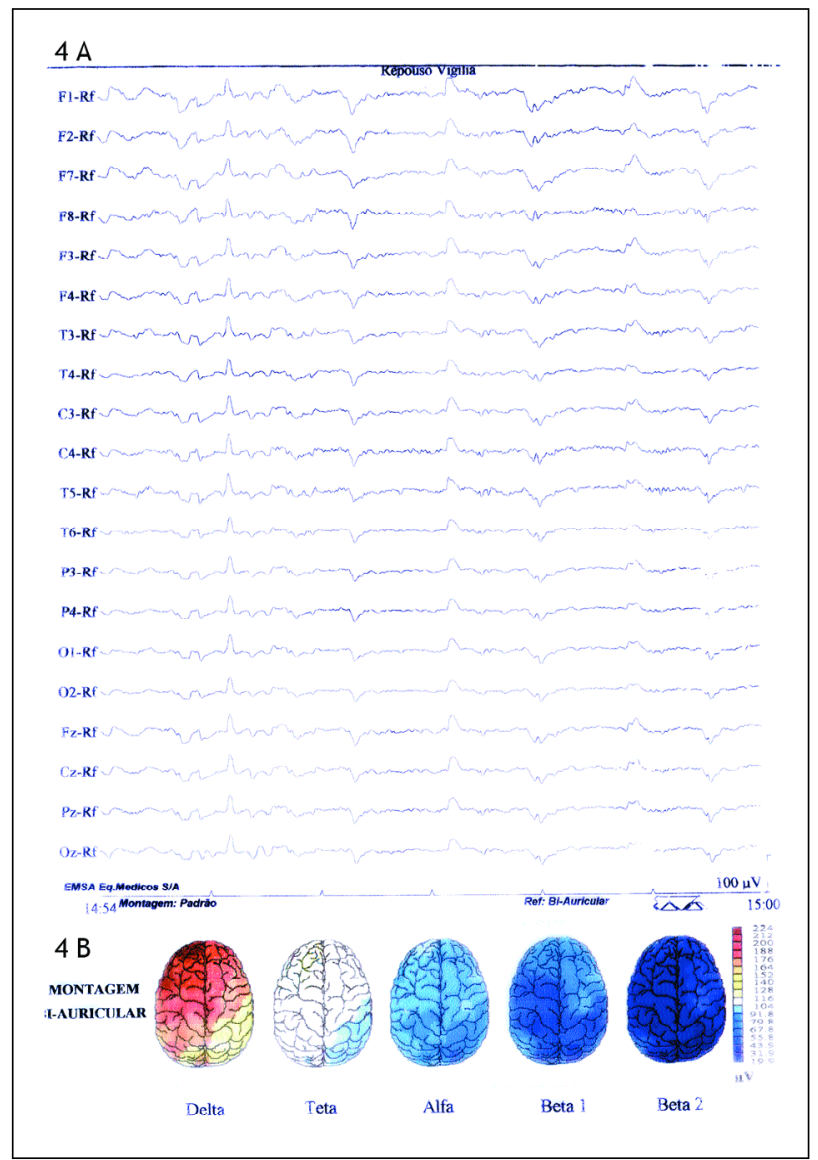

Fig 4. Movimentação de língua bilateralmente. A) análise visual do EEG: atividade lenta, irregular, ora com polaridade positiva, ora negativa, entremeada por atividade rápida de distribuição difusa. B) maior amplitude espectral na faixa de frequência delta com distribuição difusa.

muscular) com predomínio em regiões temporais anteriores e posteriores, C3-C4 / T3-T4 / F3-F / T5-T6. A quantificação destes achados mostrou predomínio das amplitudes espectrais em região temporal média bilateralmente nas faixas beta 1 (principalmente) e beta 2, com difusão para as regiões anteriores e posteriores Foi observado também aumento das amplitudes espectrais em regiões anteriores na banda desta, por vezes com predomínio em um dos hemisférios cerebrais (Fig 3).

Movimentação de língua bilateralmente - A análise visual mostrou uma atividade lenta, irregular, ora com polaridade positiva, ora negativa, entremeada por atividade rápida de distribuição difusa. $A$ análise quantitativa mostrou predomínio espectral na faixa de frequência delta com distribuição difusa e maior em região média e anterior, bilateralmente (Fig 4). 


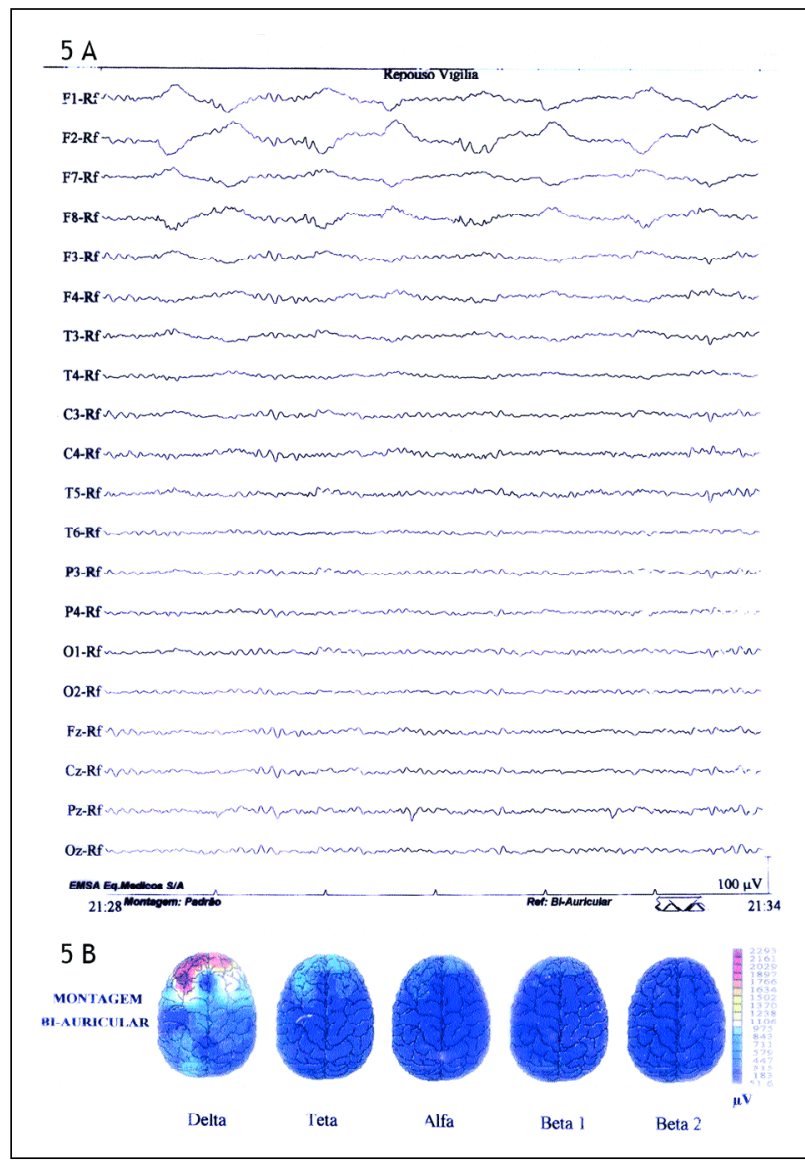

Fig 5. Movimento ocular em varredura horizontal. A) análise visual do EEG: atividade lenta anterior com reversão positiva em Fp1-Fp2 e Fp7-Fp8. B) análise espectral: maior amplitude espectral anterior bilateral, na banda delta.

Movimento ocular em varredura horizontal - A análise visual de EEG mostra uma atividade lenta anterior com reversão positiva em Fp1-Fp2 e Fp7-Fp8. A análise quantitativa mostrou amplitude espectral com predomínio anterior bilateral, na faixa de frequência desta (Fig 5).

\section{DISCUSSÃO}

Dos artefatos de EEG, um dos mais freqüentes é o de piscamento palpebral. O padrão encontrado à análise quantitativa do EEG é congruente com os achados da análise visual. Portanto, quando observamos à análise espectral potenciais de alta amplitude com marcante predomínio anterior, é importante obsenarmos novamente o traçado para verificar se este padrão não tem características próprias de um artefato. Outro achado de nosso estudo mostrou padrão semelhante ao do piscamento palpebral à análise quantitativa, que foi o artefato por movimento ocular em varredura. A diferenciação entre ambos só será possível com a análise visual do traçado, onde o padrão de piscamento palpebral apresenta ondas lentas em concordância de fase em eletrodos anteriores e o movimento de varredura apresenta padrão de discordância de fase nos mesmos eletrodos.

$\mathrm{O}$ artefato por fechamento dos olhos apresenta dois picos de amplitudes espectrais. O primeiro na banda de frequência alfa e predomínio posterior. Este corresponde a atividade alfa fisiológica e o segundo de padrão beta 1 anterior, este sim, correspondendo ao padrão muscular (beta) palpebral em eletrodos anteriores do escalpo.

O fechamento forçado da mandíbula mostrou um padrão beta 1 e beta 2 com maior amplitude em regiões temporais, padrão já classicamente encontrado no EEG com análise visual.

O movimento de língua em varredura mostra um padrão à análise visual com certa periodicidade e distribuição difusa, re presentado à análise espectral por um potencial delta difuso.

Em conclusão, é fundamental que a análise visual do EEG seja realizada por um eletrencefalografista capacitado, que possa identificar os seguimentos do traçado sempre que possível livres de artefatos, escoIhendo-os para o processamento da transformada rápida de Furrier (FFT). É recomendado que algumas medidas sejam praticadas durante a aquisição do exame, no intuito de monitorar a ocorrência de artefatos, tais como a colocação de eletrodo que monitore o movimento ocular e palpebral e a captação de um t recho do exame com o paciente com os olhos abertos, o que abole ou ao menos minimiza o piscamento palpebral. O relaxamento do paciente, e a sua orientação durante o exame, podem ajudar a diminuir a o co rrência de artefatos palpebrais, oculares e musculares. Contudo, mesmo que todos estes cuidados sejam tomados os resultados da análise quantitativa do EEG podem manter-se com significado incerto, devido a artefatos ou ruídos que contaminem o regist ro ${ }^{14}$. Portanto, o conhecimento do comportamento dos artefatos quando representados cartograficamente no EEG é de suma importância, para alertar o observador a retornar à leitura visual do traçado, no intuito de certificarse que aquele potencial encontrado na análise espectral não seja interpretado como patológico. Fica evidente que a análise visual de EEG é superior à quantitativa para o estudo de a rtefatos, por ser a mesma feita no domínio do tempo, respeitando o estudo morfológico dos paroxismos no registro, características que são perdidas no estudo de espectro (domínio de frequência). 


\section{REFERÊNCIAS}

1. Markand, ON. Artifacts. In Annual meeting of American Academy of Neurology 1996;1:363-396.

2. Jerwis BW, Nichols MJ, Allen EM, Hudson NR, Johnson TE. The assessment of the two methods for removing eye movement artifact from the EEG. Electroencephalogr Clin Neurophysiol 1985;61:444-452.

3. Duffy FH, lyer VG, Surwillo WW. Clinical electroencephalography and topographic brain mapping. New York: Springer Verlag, 1989: 99-132

4. Mau rer K, Dierks T. Atlas of brain mapping. Berlin: Springer Verlag, 1991:41-44.

5. Delamonica EA. Electroencefalografia. Buenos Aires: Ateneo 1975:62-86.

6. Nuwer MR. Quantitative EEG analysis in clinical settings. Brain Topogr 1996;8:201-208.

7. Luccas FJC, Braga NIO, Fonseca LC, Frochtengarten ML. Recomendações para o registro e interpretação do mapeamento topográfico do EEG e PES: Parte I. Aspectos gerais. Braz J Epilep Clin Neurophysiol 1996;2:175-182.

8. Joyce CA, Gorodnitsky IF, Kutas M. Automatic removal of eye movement and blink artifacts from EEG data using blind component separation. Psychophysiology 2004;41:313-325.
9. Vanhatalo S, Voipio J, Dewarwja A, Holmes MD, Miller JW. Topography and elimination of slow EEG responses related to tongue movements. Neuroimage 2003;20:1419-1423.

10. Lesser RP. Guidelines Committees of American Electroencephalographic Society. J Clin Neurophysiol 1984;11:9-11.

11. Nuwer MR. Assesment of digital EEG, quantitative EEG and EEG brain mapping: report of the American Academy of Neurology and the American Clinical Neurophysiology Society. Neurology 1997;49: 277-292.

12. Epstein CM. Digital EEG: choosing the right equipment for your lab. In $30^{\text {th }}$ Annual course in clinical EEG and Electrophisiology of American EEG Society. Washington, D.C. American EEG Society / Executive OfficePO. Bloomfield, CT:1995.

13. Jasper HH. The ten-twenty electrode system of the Internacional Federation. Electroencephalogr Clin Neurophysiol 1958;10:371-373.

14. Lagerlund TD, Worrell GA. EEG source localization (Model-Dependent and Model- Independent Methods). In Niedermeyer E, Silva FL (eds). Electroencephalography: basic principles, clinical applications, and related fields, $5^{\text {th }}$ ed, 2005:829-844. 